\title{
Analysing DNA patents in relation with diagnostic genetic testing
}

\author{
Birgit Verbeure $^{1,2}$, Gert Matthijs ${ }^{*, 2}$ and Geertrui Van Overwalle ${ }^{1}$ \\ ${ }^{1}$ Centre for Intellectual Property Rights, Faculty of Law, Catholic University of Leuven, Leuven, Belgium; ${ }^{2}$ Department \\ of Human Genetics, Faculty of Medicine, Catholic University of Leuven, Leuven, Belgium
}

In the ongoing debate concerning DNA patents, there is a need for empirical data. We aim at creating this data set for DNA patents related to diagnostic genetic testing. To this end we developed two tools to facilitate this process. First, we set up a search strategy to find the relevant patents. Second, we provide a claim classification template to assist the user in the assessment of the subject matter covered by the patent claims and in creating a comprehensive overview of the patent situation within this field. These tools have been used in a pilot study on 11 selected hereditary disorders. In addition, a detailed analysis of the familial breast and ovarian cancer genes patents retrieved by the developed search strategy and their claim classification, after meticulous reading of the documents, allowed us to better describe the problems which medical geneticists and researchers might face when dealing with the patented technology.

European Journal of Human Genetics (2006) 14, 26-33. doi:10.1038/sj.ejhg.5201503; published online 12 October 2005

Keywords: gene patents; diagnostic testing; breast cancer genes; BRCA1; BRCA2

\section{Introduction}

The appropriateness of patenting DNA sequences and genetic technologies is, still, a matter of debate and controversy. Initially, DNA patents were mainly focused on newly cloned genes encoding therapeutic proteins, for example, human t-Pa (EP0093619) or human insulin (EP0055945). Hence, in this area of research and in the development of therapeutics, gene patents were considered equivalent to patents on new chemical entities with a therapeutic use where patenting was accepted as an established management strategy. But underlying science advanced. Newer and faster tools and techniques became available for identifying genes and their involvement in diseases. Today, genetic sequence information no longer has its main application in recombinant technology and the supply of therapeutics, but the data are used in the much broader context of life science research, drug

*Correspondence: Dr G Matthijs, Department of Human Genetics, University of Leuven, Herestraat 49, B-3000 Leuven, Belgium.

Tel: + 3216 346070; Fax: + 3216 346060;

E-mail: Gert.Matthijs@med.kuleuven.be

Received 4 March 2005; revised 16 August 2005; accepted 26 August 2005; published online 12 October 2005 development, diagnostics, etc. ${ }^{1}$ This evolution generated patents that play different roles in management strategies in the biomedical community: the scope of patents on DNA sequences evolved from patents on gene-constructs encoding therapeutic proteins, to patents on DNA sequences including not only their therapeutic utility in encoding the protein, but also the application of the knowledge regarding a gene sequence in diagnosis and research. Therefore, the interests at stake and the group of professionals affected changed and set off a delayed but persistent concern about patenting genes and the approach to be taken in this field. ${ }^{2}$

Regarding diagnostics, several studies have been published on the possible influence of patents and licensing strategies on the provision of clinical genetic testing services. ${ }^{3-8}$ The main focus has been on analyzing licensing practices and understanding the strategies of companies and research organizations in their attempt to exploit acquired rights over their inventions. Publications mostly result from surveys held with companies, research centers and diagnostic service providers, both public and private. ${ }^{9,10}$ Two major concerns have been put forward: first, clinical geneticists feel that the service towards 
patients is hampered, and second, research and new test development may be inhibited by patents. ${ }^{11-13}$

Despite the value of these studies by illustrating an important issue in the relation between patenting and (public) health services, no in-depth data are available yet on the scope and effective characteristics of the problem described. Most of the reports contain only anecdotal evidence to support their conclusions, probably because broadly based evidence is not readily available. Additionally, the reports available to a large extend focus on ownership and licensing practices and not necessarily on the patents and the scope of patent protection. Only a few papers discuss the patentability itself, whereby a recent American study questioned the patentability requirements for DNA sequences in detail. ${ }^{14}$ In any case, the emphasis in the published surveys lies with the patent situation in the US, Canada and Australia. We aim at creating a better understanding of DNA-patents and the actual protection that they confer by the wording of the claims, while focusing on the European situation. Our methodology is based on gathering empirical data by collecting and studying in an exhaustive way the patents that are of importance for genetic diagnostic testing. In this way, a valuable tool and an empirical basis for policy development are created. At the same time, the developed tools could assist those working in the field of diagnostic testing to find and read patents related to their specific scientific needs.

\section{Results and discussion}

Gathering the empirical data consists of two aspects for which tools were developed. On the one hand, the patents relating to genetic diagnostic testing have been searched in an existing database. On the other hand, after careful reading of the resulting patents, the claims were analyzed in detail and classified. The presented search and classification tools were formed gradually throughout and were applied in a pilot study that was performed on a select number of genetic disorders and the genes involved.

\section{The patent search}

The number of patents filed and granted at the European Patent Office (EPO) is large. For example, 5474 European patent documents in the field of biochemistry and genetic engineering (international patent class C12) were published in 2004. It is therefore imperative to use a good search strategy in order to find the relevant documents.

Different patent databases were tested in order to set up a robust search strategy and to find the patents relevant to this area of practice. A more complete list of patent resources can be found on the EPO website (http://www. european-patent-office.org/online/index.htm\#databases). A main distinction can be made between the noncommercial and freely accessible databases of which most are supported by patent offices, and the fee-based commercial databases. A key example of a noncommercial database is Espacenet ${ }^{\circledR} \quad($ http://ep.espacenet.com/), a patent resource from the EPO. This database is freely accessible and comprises a worldwide patent collection. Unfortunately, in Espacenet ${ }^{\circledR}$ only a limited number of search terms is accepted by the search engine and text is only searchable on title and abstract. The United States Patent and Trademark Office (USPTO) also offers a freely accessible on-line database which unfortunately is limited to US patents and patent applications, although the documents are full-text searchable (http://www.uspto. gov/patft/index.html). Commercial databases such as Delphion ${ }^{\circledR}$ (http://www.delphion.com/), STN International ${ }^{\circledR}$ (http://www.stn-international.de/), Dialog ${ }^{\circledR}$ (http://www. dialog.com/) or Micropatent ${ }^{\circledR}$ (http://www.micropatent.com/ static/index.htm) are supported by a more performing search platform, thereby responding to the need of patent professionals for the use of more complex search algorithms.

Nevertheless, finding the relevant patents might remain a cumbersome undertaking. Already several initiatives led to the construction of 'subset patent databases'. These subset databases offer a collection of patents limited to a certain technological area. Several of these databases focus on biotech patents and aim to assist both professionals and nonprofessionals to understand and navigate the biotech patent landscape. An interesting example is the DNA Patent Database (DPD, http://dnapatents.georgetown.edu). DPD is a joint project of Georgetown University's Kennedy Institute of Ethics and the Foundation for Genetic Medicine. The DPD contains DNA patents issued by the USPTO. DPD is created to make full-text patents available at no cost and to define a searchable set of patents of interest to those studying genomics, genetics, biotechnology and other fields. Another example is the BiOS patent database for life sciences (http://www.bios.net) that contains patents relating to a rather broad area of life sciences, covering biology, biotechnology, medicine, chemistry, agriculture, food science, etc. The BiOS collection currently consists of life science patents from the US, Australia and Europe and contains a subset of all patents, extracted on the basis of the International Patent Classification (IPC) codes pertaining to the life sciences encompassing a rather broad range. All patents in the life sciences, impacting public health, medicine, pharmaceuticals, chemistry, environmental management and genetic resources, as well as food, nutrition, agriculture and biotechnology are taken up in this database. Both these subset databases - DPD and BiOS - did not exactly correspond to our needs for identifying patents defined to the field of diagnostics but were very helpful in the development of our search strategy.

We developed a patent search strategy that runs in two steps. At the first stage, a general search algorithm was 
constructed. The aim was to obtain a first collection containing essentially all DNA-patents relating to genetic diagnostic testing while reducing the amount of interfering nonrelevant patents. The general search algorithm set up for this study is similar to the search algorithm underlying the DPD. Patents included in the DPD were identified by virtue of their USPTO classification codes and the presence of keywords in the claims such as 'DNA'. This combination of USPTO classification codes and keywords is called the Cook-Deegan algorithm developed by Robert CookDeegan at Duke University.

By reducing the noise, this first selection greatly facilitates the search in the second stage: the search for diagnostic DNA patents on a specific gene or hereditary disorder. Indeed, we were interested in a subset of the collection gathered for the DPD, namely patents concerning the information relevant to diagnostic testing only. Also, because our primary focus is the situation in Europe, we based our search on the relevant IPC codes instead of DPD's combination of USPTO classification codes. The BiOS patent database, although set up on the basis of IPC codes, does not offer an advantage over the conventional databases either since it encompasses a selection that is too broad when considering DNA patents related to diagnostic genetic testing only.

For the selection of the IPC codes for our search algorithm, the major hurdle to overcome was that gene or DNA patents do not coincide with a specific IPC category. Therefore, a group of IPC codes has been selected encompassing the various IPC codes that have been given by the patent offices to the DNA patents that we aim to retain by our search (see Table 1 ). The combination of IPC codes in our search algorithm is the result of an iterative process which we performed, starting from a group of codes attributed to a set of manually searched patents relevant to our goal. This initial list has been narrowed down on the basis of our own analysis of the patents and our insight in the field of diagnostics, in a way to reduce the amount of nonrelevant patents without loosing the intended documents in the result of the search.

The group of patents thus obtained was then further limited by a keyword search string in the claims. For the keyword selection, a similar process was conducted as for the IPC code selection, starting from the list of keywords used in the Cook-Deegan algorithm. The resulting algorithm that we used for a first selection of patents, combines IPC codes and keywords appearing in the claims as shown in Table 1. The search algorithm was set up in the commercial database of Micropatent ${ }^{\circledR}$. Unfortunately, the available free of charge patent databases such as Espacenet ${ }^{\circledR}$ are not equipped with a search engine that allows the degree of complexity of the developed algorithm.

The resulting collection of DNA patents was used in the next step of our search: finding DNA patents relevant to genetic diagnostic testing on a specific gene or for a specific hereditary disorder. To this end, the collection has been searched on the basis of keywords specifically associated with the gene (name of the gene and gene product and the relevant synonyms, the gene's letter code etc.) and/or the disorder related to the gene. For example, for Huntington's Disease the keyword selection included huntington, huntingtin, 'HD' and 'IT15'.

In a pilot study, a set of hereditary disorders was used to optimize and test the developed search strategy. The disorders included in this test, together with the relevant genes searched and patent entries found, are listed in Table 2. They represent key examples selected on criteria such as disease frequency, inheritance pattern, frequency in the population, clinical importance, types of testing available (diagnostic, predictive, prenatal), availability of alternative testing techniques, and whether or not treatment is available. Patents were selected in function of their relevance to genetic diagnostic testing. In practice, essentially all patents that could affect genetic diagnostic testing based on the cited genes were selected from the search results list. This includes patents with product claims on the gene itself or on mutated forms, as well as on diagnostic methods or kits involving the gene. Although emphasis of this study is put on the European patent situation, US patents were included for comparison.

Apparent from this pilot study is the difference in number of patents issued at the present time in Europe compared to the US: in general more US than EP patent documents are retrieved. At least three reasons can be put

Table 1 General search algorithm for DNA patents relevant to diagnostics

(IPC: C07H021* OR C07K014* OR C12N0151* OR C12N0152* OR C12N0153* OR C12N0154* OR C12N0155* OR C12N0156* OR C12Q00168 OR G01N0335* OR G01N0336*) and (keywords in claims: 'gene' or 'genes' or genetic or genomic or genotype or haplotype or DNA or DNAs or CDNA* or RNA or RNAs or mRNA* or 'nucleic acid' or 'nucleotide sequence' or polynucleotide or exon or exons or intron or introns or probe or probes or primer or primers or hybridisation or hybridization or polymorphi* or marker or mutation* or mutant or mutated or allelic or allele or 'wild type' or substitution or deletion or insertion or alteration or diagnos* or predisposition or susceptibility)

The search results in a collection of patent documents classified according to one of the IPC codes listed in the algorithm, and containing at least one of the listed keywords in the claims.

* = Wild card character (no limit on characters added, eg, polymorphi* encompasses polymorphic, polymorphism etc);

$' \ldots{ }^{\prime}=$ Exact phrasing only. The definition of IPC codes used in this search algorithm (C07H 21, C07K 14, C12N 15, C12Q 01/68, G01N 33) can be found on http://www.wipo.int/classifications/fulltext/new_ipc/index.htm. 
Table 2 Listing of a set of hereditary diseases and their genes (as indicated on Genetest website and OMIM entries) taken up in the pilot study

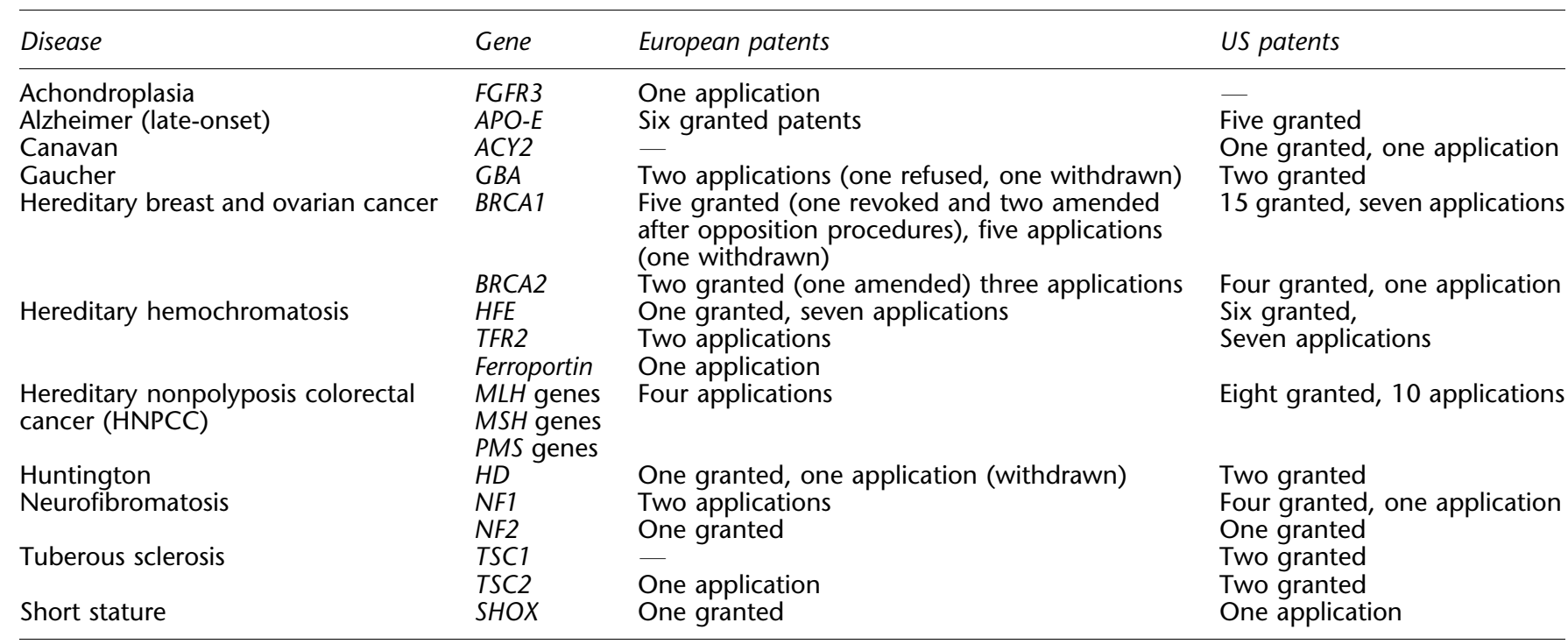

The patent count includes all patents that could affect genetic diagnostic testing based on the cited genes thus including patents with product claims on the gene itself or mutated forms, as well as diagnostic methods or kits involving the gene (last updated 16 August 2005).

forward to explain this difference. Part of this phenomenon has been attributed to a backlog in the granting procedure at the EPO, ${ }^{15}$ so that a lot of the applications are still pending (eg Hereditary Hemochromatosis, Hereditary Non-Polyposis Colorectal Cancer (HNPCC)). Alternatively, it has been indicated that the EPO would employ higher standards than the USPTO although no data effectively substantiated this statement. We also noticed that in some of these cases either the inventors applied for a patent in the US but not in Europe (eg TSC1, ACY2), or some of the EP patent applications of which the US counterpart has been granted, have been refused or withdrawn from the European procedure (eg $H D, G B A$ ), or revoked after opposition (BRCA1, first instance, the patent proprietors filed an appeal against the decision) (Table 2).

Another important remark resulting from this pilot study is that one also has to be cautious in focusing on the number of patents resulting from a search. Differences in patent law between the US and Europe can result in a different number of patents, covering the same subject matter. An important reason for this is for example that a product claim on the nucleic acid sequence and a product claim on a protein sequence can occur in the same EP patent, whereas in the US two different patents have to be filed for each type of product claim.

\section{Patent characterization}

Subsequent to the collection of relevant patents, we gathered additional information with regard to the type of claims. The claims of a patent define the scope of protection conferred by the patent. As already indicated above, some of the collected DNA patents are more fundamental than others. For example, a patent covering the full cDNA sequence as a product is more fundamental than a patent disclosing a method for using this sequence in a diagnostic method. In the report of the Nuffield Council of Bioethics, ${ }^{8}$ serious concern was raised on the granting of inappropriately broad patents where the actual utility disclosed in the patent only relates to a specific application or use of the claimed subject matter: 'protection is sought primarily for DNA sequences as such and extended with their application in method or use-claims'. Within the variety of possible applications of genetics, in terms of patent protection, distinction has been made in four main method or use categories: the production of therapeutic proteins, diagnostic methods, research tools and gene therapy. Patents cast in broad terms of the gene sequence as such, effectively give the patent holder the exclusive right to control all downstream uses of the sequence, including research and development of tests, therapy and a whole range of diagnostics. The actual wording of the claims in those patents can have important bearings on the effect patents have both on health care services and on future research and innovation. It is therefore imperative to have actual data on the types of claims that have been granted to assess the breath of the assumed problem.

To this end, we have designed a detailed template to classify the claims after a thorough analysis of the granted European patents. The result is a detailed overview of the different types of product, method and use claims. This classification of claims will help in creating a better general understanding of the scope of protection conferred by the 
DNA patents granted. Within the three main claim types, subdivisions were made as listed in Table 3, panel A. A direct comparison of claim classifications of different patents will also visualize where interferences between different patents might exist and hence where a conflict of interest between different patent proprietors might occur.

The template could assist the user when trying to assess what subject matter is covered by the patent claims. It presents a comprehensive overview of patented materials, methods or uses regarding a certain gene sequence. The template could also be useful for researchers and other professionals in the field of genetics, to whom the patent language can be obscure and confusing, to help them in a useful direction. The classification might thus help 'to render the massive, complex and opaque world of patents and IP into a transparent and stimulating structure for the public good, as originally intended by framers of patent systems' (citation from www.bios.net), not only at the level of patent search but also at the level of understanding the scope of protection. Although the claims of the patents have been read carefully and in light of the specification for their classification in the template, one still needs to read the patent itself to know exactly what is covered by the claims. This is inevitable because of the importance of the wording of the claims and of the support for those claims that has to be found in the patent specification.

We have illustrated the utility of the classification template by applying it to the patents on the familial breast and ovarian cancer genes BRCA1 and BRCA2 (see Table 3, panel B). Oppositions were filed at the EPO against three of the patents related to BRCA1. This resulted in one patent being revoked (EP0699754) ${ }^{16}$ and two patents upheld in amended form (EP0705902 and EP0705903) ${ }^{17}$ (decision for EP0699754 and minutes of the oral proceedings for EP0705902 and EP0705903 are available for download at the EPO's Online Public File Inspection on http://ofi.epoline.org/view/GetDossier). For the purpose of comparison and for its illustrative value on the gene patenting issue, the set of claims as originally granted as well as the amended set of claims for these patents are retained in this study. Meanwhile, the proprietors of the patent EP0699754 filed appeal against the decision to revoke. Oppositions have also been filed against the BRCA2 patents. EP0785216 has been upheld in amended form after recent proceedings at the EPO. ${ }^{18}$ The breast cancer genes' patent situation has been amply commented on in the recent past and left a tumultuous trail throughout the research, medical and patent law community. Nevertheless, a comparative and in-depth analysis of all the granted patents to date and the scope of their claims has not been reported in the literature. We have tried through this claim analysis to further clarify the situation under debate.

As can be read from the claim classification templates for both BRCA1 and BRCA2 (Table 3), inventors aim at claims in the product category that cover the full cDNA sequence (line I.2) as a prime objective due to the broad protection it confers. Methods related to diagnosis (line II.1-6) constitute another main category, ranging from a claim covering the determination of a variation in the cDNA sequence (deleterious or not, lines II.1 and 2), or more specifically by claiming diagnostic methods based on the cDNA sequence and identified/disclosed deleterious mutations (line II.5). A last major group of claims comprises methods or products covering the therapeutic application of the knowledge that stems from the genetic sequence in gene therapy or recombinant production of the protein for therapeutic purposes (lines I.19, I.21-27, II.9, III.1-3 and III.7-8).

Products that are not perceived as having that much direct commercial utility, seem to get much less coverage despite their importance for more fundamental research purposes. This seems to be the case for the genomic sequence (line I.5). Besides possibly the unavailability of the genomic sequence at the time of the invention, this may also be due to a lack of interest from a commercial point of view at that time. Only a minor percentage of deleterious mutations were found in intronic parts of the gene and hence it was uncertain whether it was worth the financial effort and the strategic risk in waiting for the genomic sequence before filing the patent. Another argument for patenting the cDNA sequence is patent technical in nature and is the non-natural character of that sequence. The generation of a cDNA sequence implies a process of isolation and purification since cDNA does not occur in nature as a DNA molecule. Support for this theory can be found in the Rule 23c(a) European Patent Convention and Article 5 and Recital 22 EU Directive 98/44/EC.

Besides the cDNA sequence, claims frequently cover fragments of the cDNA sequence (lines I.7 and 8). In some cases, this may result from the fact that these fragments were the only actual sequence data available to the inventors for disclosure in the patent application at the time of filing. This was the case with one of the BRCA2 patents (EP0858467). In most other cases, cDNA fragments are usually claimed in terms of necessary tools for diagnostic and therapeutic purposes, for example, primers for PCR amplification, (labeled) probes for mutation detection etc.

This distribution of the subject matter illustrates that the scope of protection sought by the inventors, clearly reflects the economic incentive to file patents. Indeed, research on the BRCA1 and BRCA2 genes was spurred by their involvement in the etiology of breast and ovarian cancer, consequently by their utility in carrier identification and diagnosis through genetic testing, and ultimately, by the prospect of developing gene specific disease therapy for example, through gene therapy or production of therapeutic proteins using recombinant technology. It is therefore not surprising that patent protection is sought in those areas of possible commercialization on a larger scale: diagnostics and therapeutics. 
Table 3 Classification template for the subject matter covered by the claims with application to the BRCA1 and BRCA2 patents (A): Different categories of claims covering product, method or use claims. (B): classification of European patents related to the BRCA1 and BRCA2 gene

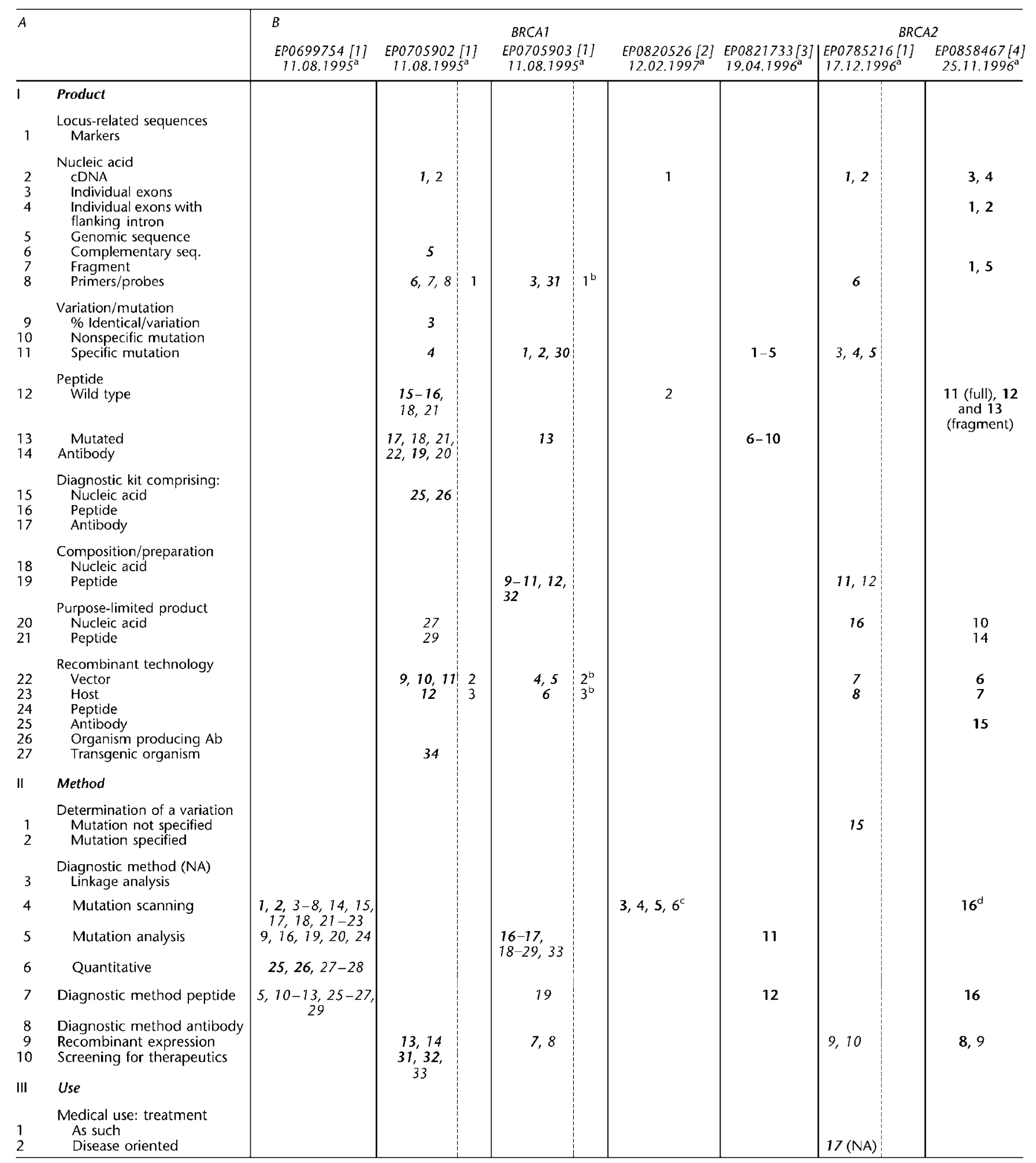


Table 3 Continued.

\begin{tabular}{|c|c|c|c|c|c|c|c|c|c|}
\hline$A$ & & $\begin{array}{l}B \\
E P 0699754[1] \\
11.08 .1995^{\mathrm{a}}\end{array}$ & $\begin{array}{c}\text { EP0705902 [1] } \\
11.08 .1995^{\mathrm{a}}\end{array}$ & $\begin{array}{l}\text { BRCA1 } \\
\text { EPO705903 [1] } \\
11.08 .1995^{\mathrm{a}}\end{array}$ & $\begin{array}{c}\text { EP0820526 [2] } \\
12.02 .1997^{\mathrm{a}}\end{array}$ & $\begin{array}{c}\text { EP0821733[3] } \\
19.04 .1996^{\mathrm{a}}\end{array}$ & $\begin{array}{l}\text { EP0785216 } \\
17.12 .1996\end{array}$ & $\begin{array}{l}B R C \\
6[1] \\
6^{\mathrm{a}}\end{array}$ & $\begin{array}{l}\text { CA2 } \\
\text { EP0858467[4] } \\
25.11 .1996^{\mathrm{a}}\end{array}$ \\
\hline 3 & Method oriented & & $\begin{array}{l}28 \text { (NA) } \\
30 \text { (pept) }\end{array}$ & & $\begin{array}{l}7 \text { (NA) } \\
8 \text { (pept) }\end{array}$ & & 78 (pept) & & \\
\hline $\begin{array}{l}4 \\
5 \\
6\end{array}$ & $\begin{array}{l}\text { Medical use: diagnostic } \\
\text { As such } \\
\text { Disease oriented } \\
\text { Method oriented }\end{array}$ & & . & & & & . & 1 & \\
\hline $\begin{array}{l}7 \\
8\end{array}$ & $\begin{array}{l}\text { Use in recombinant technology } \\
\text { Of nucleic acid } \\
\text { Of peptide }\end{array}$ & & 23,24 & 14,15 & & & 13,14 & & \\
\hline
\end{tabular}

Independent claims are in bold. Claims in the patents as originally granted but revoked or amended during opposition procedures at the EPO are in italic and separated from the current enforceable set of claims by a dotted line. [1] The patent has been jointly filed by Myriad Genetics, the University of Utah Research Foundation and the United States of America. Recently, Myriad Genetics transferred its rights on the invention in Europe over to the University of Utah Research Foundation; [2] The patent was originally filed by Oncormed that has been taken over by Gene Logic; [3] The patent proprietor is the Regents of the University of California; [4] the patent was originally filed by Cancer Research Campaign Technology Limited and Duke University, the former merged with the Imperial Cancer Research Fund and formed Cancer Research UK.

${ }^{\mathrm{a}}$ Date of filing the patent application.

${ }^{\mathrm{b}}$ Claim refers to one specific mutation only (185delAG).

${ }^{c}$ Claim disclaims diagnostic methods determining mutations known in prior art.

${ }^{\mathrm{d} C l a i m}$ covers mutation scanning in part of the BRCA2 gene only.

(NA): method or use based on the nucleic acid, (pept): method or use based on the peptide.

Not only the profiling of the patented subject matter in these gene patents in general, but also the comparative analysis between patents in the same field is interesting. It is striking that the cDNA sequences of both BRCA1 and $B R C A 2$ (line I.2, BRCA1 claims before amendment during opposition procedures) seem to be covered by two different patent families and according to our information, to be owned by two different proprietors. To know how and why this was possible, one has to look at the claims themselves. For example, both patents on the BRCA2 gene originally claimed a cDNA sequence. After a long and cumbersome examination procedure (see examination procedure at the EPO, available online at http://ofi.epoline.org/view/Get Dossier), a patent (EP0858467) for the full BRCA2 coding sequence encompassing all allelic variants was granted through a product-by-process claim (line I.2). The inventors eventually got awarded the full coding sequence of the different allelic variants by disclosing already in their priority documents part of the sequence and methods using the sequence data and thereby teaching the person skilled in the art to arrive at the full sequence. Another application resulted in the grant of a downstream patent (EP0785216) with protection for a single allelic variant by disclosing the actual full coding sequence of that BRCA2 allele. Looking further into the method claims, the situation got more complicated. On the one hand, EP0785216 empowered the rights for a method for determining any variation in the full $B R C A 2$ cDNA sequence but in reference to the cDNA sequence of one specific allele only (line II.1). On the other hand, EP0858467 entitles the owner to the rights for diagnostic testing on BRCA2 in reference to all its allelic variants but not on the full-length cDNA sequence (EP0858467 line II.4). Thus, despite the fact that the EP0858467 proprietors hold the primary rights over the full-length cDNA sequence, for their diagnostic claim they may only refer to the part of the wild-type BRCA2 cDNA (approximately $70 \%$ of the full cDNA) of which they effectively disclosed the sequence in their second priority document (GB9525555).

Before the amendment of the claims of EP0705902 during the opposition proceedings at the EPO last January, a similar situation characterized the BRCA1 patent landscape. Claim 1 of the patent with the earliest filing date (EP0705902) was drafted in a way to encompass all allelic variants possibly coding for a BRCA1 polypeptide. The main cDNA claim in the later filed patent EP0820526 covers only one possible coding sequence. The fact that the latter sequence is a consensus sequence (corresponding to only one be it the most frequently occurring allele) was considered to be the special and unexpected feature of this specific cDNA sequence, and considered as a further advancement in the technological field. This situation led to a conflict of interest between the different proprietors. ${ }^{19}$

The above analysis already illustrates how one patent does not necessarily preclude further patenting within a technological field, but allows further advancements and refinements to the state of the art to be rewarded by patents as well. Hence, the existence of a patent application or a granted patent on the general technological feature in the field, the full cDNA sequence, does not preclude opportunities for innovation. Although a patent application is not enforceable before grant, its existence 
implies the risk that future developments will be infringing once granted. At the same time, the principle of 'protection for disclosure' to stimulate innovation, a founding incentive for the patent system, seems to work in this technological field. An upstream patent covering the cDNA sequences leaves the possibility for others to file patent applications for new uses of the patented product, and even for new methods for producing the products, under the provision that the patent holder of the product patent could not have foreseen the new process or method. For genetic testing purposes, a key example is the patenting of newly identified mutations. For both the BRCA1 and $B R C A 2$ gene, various patent applications have been filed claiming newly identified mutations, and in the case of BRCA1, one such patent has recently been granted (see Table 3, EP0821733, line I.11). However, the fact that this might be patentable subject matter does not automatically allow the inventor to exploit his invention. By carrying out this invention one could be infringing an upstream patent. Besides their potential impact on further research and innovation, the powerful position of such upstream patents vis-à-vis the genetic testing practice thus remains.

\section{Conclusion}

In our research program we aim at creating an empirical data set for DNA-patents related to diagnostic genetic testing. We developed two tools to facilitate this process. First, we set up a search strategy to find the relevant patents. Second, we provide a claim-classification template to enable an immediate comprehensive overview of the patent situation for topics - genes or diseases - within this field. The claim classification brings sought-after added value to the collection of patents in support of the study of the legal framework. Apart from the controversy on the patenting of DNA, this classification of the claims related to the BRCA1 and BRCA2 genes nicely illustrates that patents in a research intensive technological field may well lead to a complicated situation causing difficulties in interpretation for third parties to know who effectively does own which rights and for which activities a license should be obtained.

The empirical data should be analyzed in view of the current legal framework on the patenting of human genes, gene products and diagnostic methods. Continuous care should be taken to confer a justifiable scope of protection to gene patents. Accordingly, the issue is not necessarily whether or not gene patents as such are justified but the way these patents are enforced and used in society, thereby taking into account both an appropriate award for the innovator as well as a guaranteed access to state of the art public health services for all. Due to the recent events, there is a strong feeling of breach of the implicit social contract comprised in the patenting system that needs to be addressed.

Disclaimer: the contents of these pages are informational only and should not be substituted for legal advice.

\section{Acknowledgements}

This research was supported by grant number G.0120.04 of the Fund for Scientific Research (FWO, Flanders, Belgium) and the Vancraesbeeck Fund (KULeuven, Belgium). Special thanks goes to Paul Vancraesbeeck (Senior advisor CIR) and William Bird (qualified British, German and European Patent and Trademark Attorney) for helpful discussions. We also thank Sjoerd Hoekstra and Olivier Lonnoy (European Patent Office, The Hague), and Nico Deconinck (Belgian Patent Office, Brussels).

\section{References}

1 Collins FS, Green ED, Guttmacher AE, Guyer MS: A vision for the future of genomics research. Nature 2003; 422: 835-847.

2 Eisenberg RS: Why gene patenting controversy persists. Acad Med 2002; 77 (12): 1381-1387.

3 Rimmer M: Myriad Genetics: Patent Law and Genetic Testing. EIPR 2003; 25: 20-33.

4 Williams-Jones B: History of a Gene Patent: Tracing the Development and Application of Commercial BRCA-Testing. Health Law J 2002; 10: 123-146.

5 Walpole IR, Dawkins HJS, Sinden PD, O'Leary PC: Human gene patents: the possible impacts on genetic services healthcare. Med J Aus 2003; 179: 203-205

6 Merz JF, Kriss AG, Leonard DGB, Cho MK: Diagnostic testing fails the test. Nature 2002; 415: 577-579.

7 Organisation for Economic Co-operation and Development (OECD): Genetic inventions, intellectual property rights and licensing practices: evidence and policies, 2002, http://www.oecd. org/dataoecd/42/21/2491084.pdf.

8 Nuffield council on Bioethics: The ethics of patenting DNA. A discussion Paper, 2002, www.nuffieldbioethics.com.

9 Nicol D: Gene Patents and access to genetic tests. Aus Health Law Bull 2003; 11: $73-77$.

10 Straus J, Holzapfel H, Lindenmeir M: Empirical survey on 'Genetic Inventions and Patent Law'; Munich. 2002 commissioned by the Federal Ministry of Education and Research, Germany.

11 Matthijs G, Halley D: European-wide opposition against the breast cancer gene patents. Eur J Hum Gen 2002; 10: 783-784.

12 Cho MK, Illangasekare S, Weaver MA, Leonard DGB, Merz JF: Effects of patents and licenses on the provision of clinical genetic testing services. J Mol Diagnostics 2003; 5: 3-8.

13 Sevilla C, Moatti JP, Julian-Reynier C, Eisinger F, Stoppa-Lyonnet D, Bressac-de Paillerets SH: Testing for BRCA1 mutations: a costeffectiveness analysis. Eur J Hum Gen 2002; 10: 599-606.

14 Paradise J, Andrews L, Holbrook T: Patents on human genes: an analysis of scope and claims. Science 2005; 307: 1566-1567.

15 Abbott A: Pressured staff 'lose faith' in patent quality. Nature 2004; 429: 493

16 Abbott A: Clinicians win fight to overturn patent for breastcancer gene. Nature 2004; 429: 329.

17 Abbott A: Europe pares down double patents on breast-cancer gene. Nature 2005; 433: 344.

18 Abbott A: Genetic patent singles out Jewish women. Nature 2005; 436: 12 .

19 Dalpé R, Bouchard L, Houle AJ, Bédard L: Watching the race to find the breast cancer genes. Sci Technol Human Values 2003; 28: $187-216$. 